\title{
Topological transition in disordered planar matching: combinatorial arcs expansion
}

\author{
Andrey Y. Lokhov ${ }^{1}$, Olga V. Valba ${ }^{2,3}$, Sergei K. Nechaev ${ }^{1,3,4}$, \\ and Mikhail V. Tamm ${ }^{3,5}$ \\ ${ }^{1}$ Université Paris-Sud/CNRS, LPTMS, UMR8626, Bât. 100, 91405 Orsay, France, \\ ${ }^{2}$ N.N. Semenov Institute of Chemical Physics of the Russian Academy of \\ Sciences, 119991, Moscow, Russia, \\ ${ }^{3}$ Department of Applied Mathematics, National Research University Higher \\ School of Economics, 101000, Moscow, Russia, \\ ${ }^{4}$ P.N. Lebedev Physical Institute of the Russian Academy of Sciences, 119991, \\ Moscow, Russia, \\ ${ }^{5}$ Physics Department, Moscow State University, 119991, Moscow, Russia. \\ E-mail: andrey.lokhov@lptms.u-psud.fr, valbaolga@gmail.com, \\ sergei.nechaev@lptms.u-psud.fr, thumm.m@gmail.com
}

\begin{abstract}
In this paper, we investigate analytically the properties of the disordered Bernoulli model of planar matching. This model is characterized by a topological phase transition, yielding complete planar matching solutions only above a critical density threshold. We develop a combinatorial procedure of arcs expansion that explicitly takes into account the contribution of short arcs, and allows to obtain an accurate analytical estimation of the critical value by reducing the global constrained problem to a set of local ones. As an application to a toy representation of the RNA secondary structures, we suggest generalized models that incorporate a one-to-one correspondence between the contact matrix and the RNA-type sequence, thus giving sense to the notion of effective non-integer alphabets.
\end{abstract}


Topological transition in disordered planar matching: combinatorial arcs expansion 2

\section{Introduction}

The problem of optimal matching in a given set of interacting variables under specific non-local topological constraints in presence of quenched disorder is a challenging question in information theory, statistical physics, and biophysics. A particularly important case of such a global constraint is given by the requirement for the optimal matching configurations to have a planar structure. Indeed, the planar diagrams play a key role in many areas, including matrix and gauge theories [1], many-body condensed matter physics 2], quantum spin chains [3], random matrix theory [4. Another area where planar matching appears naturally is the biophysics of secondary structures of RNA molecules [5, 6, 7, 8]: the RNA molecules differ from other biologically active associating polymers, for instance proteins, by a formation of hierarchical "cactus-like" secondary structures, topologically isomorphic to a tree. In other words, the bonds between monomers can be drawn in a form of a planar diagram with non-intersecting arcs, while the configurations that do not obey this property are suppressed [9].

Matching problems have attracted considerable attention in mathematics, physics and computer science communities [10]. An equivalent dimer covering problem on planar lattices has been studied by Kasteleyn [11. Recently, the existence of a new phase transition has been reported in the problem of complete planar matching on a line [13, 14]. For a sequence of $L$ points, an instance of the problem is given by a symmetric $L \times L$ contact matrix $A$ with entries $A_{i j}$ taking values one (if matching between $i$ and $j$ is in principle possible) and zero (if a link $(i, j)$ is forbidden). The question that we are trying to answer is whether it is possible to draw a complete matching of $L / 2$ non-intersecting arcs involving all the points (see Fig. 1 (a) for an example). In [14], we assumed that the entries of the contact matrix are generated according to the simple Bernoulli model, i.e. they are independently equal to one with probability $p$, and are equal to zero otherwise. We have shown that perfect planar matching solutions follow a critical behavior: they exist only above a certain critical density of possible contacts, or unity entries in the contact matrix. Along with an accurate numerical study, two analytical estimations of the critical point have been provided, however, making use of uncontrollable, to a certain extent, approximations. The difficulty in these calculations arise essentially from the quenched nature of the disorder in the random contact matrix. One of the estimations featured the matrix model formulation suggested in [12, leading to a field theory 
with a complicated interaction in a form of infinite series that needs to be averaged out. Another was based on a combinatorial approach, explicitly accounting for the contribution of shortest arcs, observed to play the dominant role in the studied planar structures. A quantification of this contribution provided a good estimate for the transition point, however making use of a non-exact mean-field-like averaging argument.

In this paper we go further in the last direction, developing a procedure for a detailed treatment of quenched disorder at the level of shortest arcs in the complete planar matching problem. In particular, we show how to get successive estimations to the value of the transition point via arcs expansion, explicitly calculating the contributions of shortest and next-to-shortest arcs, and treating the contribution of the rest in a mean-field manner. These calculations involve a representation in terms of spin chain models, as well as combinatorial and generating functional formalism. Aiming at the application for the random RNA-type sequences, we introduce two new models involving explicit representation of an instance of the problem as a string of letters, and numerically study the phase transition of interest.

The paper is organized as follows. In section 1, we provide definition of the model and briefly report the previously established results. In section 2, we present an exact treatment of the first order in arcs expansion, mapping the problem to a spin chain model and calculating the contribution of the shortest arcs. In section 3 , we show how to generalize these computations to include the correlations arising from the next-to-shortest arcs in presence of a quenched disorder. Finally, in the section 4 we numerically study other models, allowing for an explicit representation in form of strings of letters, and investigate the effect of transitivity on the existence of the perfect-imperfect phase transition, making connections to the fluctuations-free Bernoulli limit.

\section{Background and definitions}

In this section we provide the definition of the model and recall some previously established results. 
Topological transition in disordered planar matching: combinatorial arcs expansion4

\subsection{Bernoulli model and mapping to Dyck paths}

The Bernoulli model of complete planar matching is stated as follows. An instance of the problem is given by a symmetric $L \times L$ random matrix $A$ containing zeros and ones. The upper-diagonal entries $A_{i j}$ of this matrix $(i>j)$ are independent identically distributed random variables, generated by the distribution

$$
\operatorname{Prob}\left(A_{i j}\right)=p \delta\left(A_{i j}-1\right)+(1-p) \delta\left(A_{i j}\right),
$$

where $\delta(x)=1$ for $x=0$, and $\delta(x)=0$ otherwise. In other words, each element $A_{i j}=A_{j i}$ is independently either one with the probability $p$ for any $i \neq j$, or zero otherwise. Now we take $L$ points $i=1, \ldots, L$ on a line, and draw $L / 2$ nonintersecting arcs between pairs of points allowed by the non-zero entries $A_{i j}$ such that each point is involved in one link only and the links form a planar diagram, see Fig. 1(a). If at least one such set of links exists, we say that the problem allows for the complete matching solution.

The phase transition [14] in this problem occurs as the parameter of the model, $p$, reaches a certain critical value of bond formation probability $p_{c}$. It can be equivalently thought of as a transition in a constrained satisfaction problem [15]: as the number of constraints per node, imposed by the matrix $A$, is below a certain critical value, the problem exhibits a complete matching solution, while otherwise no complete matching solution exists in the limit $L \rightarrow \infty$.

In what follows, we use an important one-to-one mapping between complete $L$-point planar diagrams and the $L$-step Brownian excursions, known as Dyck paths [16]. In this representation, also called mountain (or height) diagram, each monomers is represented by either an "up"-step $(\nearrow)$ or a "down"-step $(\searrow)$ with "up"-steps corresponding to opening arcs, and "down"-step to closing ones. An example is given in the Fig. 1, with the steps up and down at positions 2 and 9, corresponding to the arc between points 2 and 9 in the planar matching structure. The total number of Dyck paths of even length $L$ is given by a Catalan number

$$
C_{L / 2}=\frac{L !}{\left(\frac{L}{2}\right) !\left(\frac{L}{2}+1\right) !} \sim \frac{2^{L}}{L^{3 / 2}} \sqrt{\frac{2^{3}}{\pi}},
$$

where the asymptotic expression is valid for $L \gg 1$. If $p=1$ in our matching problem, all the planar configurations are solutions to the perfect matching problem, and their total number is then also given by (2). For $p<1$, the number of possible planar 
Topological transition in disordered planar matching: combinatorial arcs expansion5

(a)

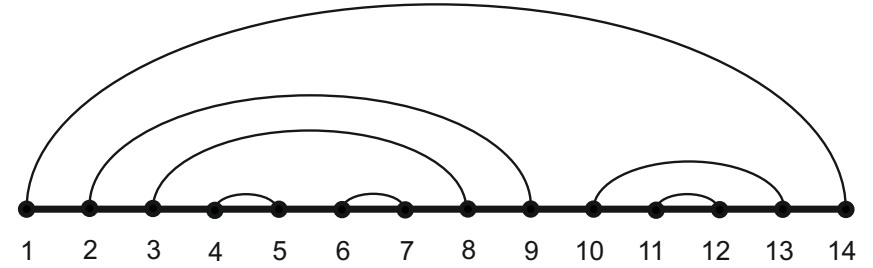

(b)

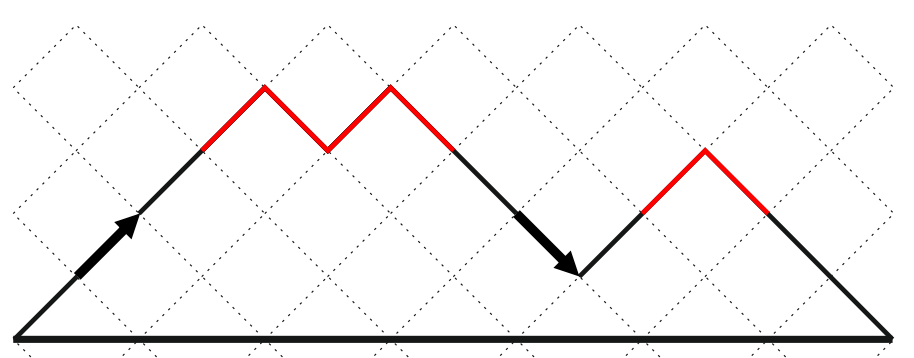

Figure 1. Example of (a) a perfect planar matching configuration, and (b) the corresponding mapping to a Dyck path. The arc is given by an "up" and "down" steps at the same height, shown by arrows $\nearrow$ and $\searrow$. The part of the walk between arrows is a Dyck path itself. The shortest arcs correspond to the peaks of the Dyck path representation and are marked with red.

configurations is reduced, and drops down to zero below a certain value $p_{c}$. A naive estimation of $p_{c}$ can be readily obtained using the following mean-field-like argument. Since each arc in the diagram is present with the probability $p$, the probability that the whole configuration is allowed, is given by $p^{L / 2}$. Assuming the planar diagrams in the ensemble of all possible ones are statistically independent, we get the probability $\mathcal{P}$ to have at least one perfect planar matching configuration:

$$
\mathcal{P}=1-\left(1-p^{L / 2}\right)^{C_{L / 2}}=1-\exp \left(-p^{L / 2} C_{L / 2}\right),
$$

where the last equality is valid for $L \rightarrow \infty$, leading to the probability one for $p>p_{c}$, and to the probability zero for $p<p_{c}$. The naive mean-field critical threshold $p_{c}$ is thus given by the condition

$$
\lim _{L \rightarrow \infty} p_{c}\left[C_{L / 2}\right]^{2 / L}=1,
$$

yielding $p_{c}=1 / 4$. However, here we have neglected the statistical correlations between different possible configurations. For instance, let $\tau$ and $\rho$ be the two 
Topological transition in disordered planar matching: combinatorial arcs expansion 6

arbitrarily chosen configuration of arcs out of $C_{L / 2}$ possible configurations. The probability that they both satisfy the constraints imposed by the contact matrix $A$ is given by $p^{L / 2} p^{L / 2} p^{-n_{\tau \cap \rho} L / 2}$, where $n_{\tau \cap \rho}$ is a fraction of common arcs in the configurations $\tau$ and $\rho$. Therefore, equation (4) provides only a crude estimation to the true value of $p_{c}$, and it has to be generalized to

$$
\lim _{L \rightarrow \infty} \xi\left(p_{c}\right)\left[C_{L / 2}\right]^{2 / L}=1, \xi\left(p_{c}\right)=1 / 4,
$$

where $\xi(p)$ is some weight (due to correlations) that has to be determined. In the sections 2 and 3, we will see how to calculate the transition value analytically in a more accurate way; before proceeding to the calculations, we present the related numerical results.

\subsection{Numerical results}

Let us briefly discuss the numerical results obtained in [14]. Finding a maximum matching on a graph is a problem of a polynomial complexity [17]. Numerically, the phase transition in the planar matching on a line can be identified using the dynamic programming algorithm, for details see [14, 18]. The idea is that the ground state free energy of the system, $F_{1, N}$, proportional to the number of nodes involved in the planar matching (and equal to $L / 2$ if the complete matching solution exists), can be computed iteratively as a zero-temperature limit of the corresponding equation for the partition function [5, 6], using the following expression:

$$
F_{i, i+k}=\max _{s=i+1, \ldots, i+k}\left[F_{i+1, i+k}, A_{i, s}+F_{i+1, s-1}+F_{s+1, i+k}\right] .
$$

Looking for the fraction, $\eta_{L}(p)$, of adjacency matrices, which allow for perfect planar matchings, in the whole ensemble of random Bernoulli matrices, one has $\eta_{\infty}(p)=1$ for $p>p_{c}$, and $\eta_{\infty}(p)=0$ for $p<p_{c}$. The finite-size results are shown in the Fig. 2 for different lengths, $L=500,1000,2000$. The phase $p>p_{c}$ corresponds to a gapless complete matching, while in the phase $p<p_{c}$ the best possible matching always contains a finite fraction of gaps. The scaling analysis places the phase transition point at $p_{c} \approx 0.379$, and allows to estimate the power-law decay of the transition width $L^{-\nu}$, with $\nu=0.5$. 
Topological transition in disordered planar matching: combinatorial arcs expansion 7

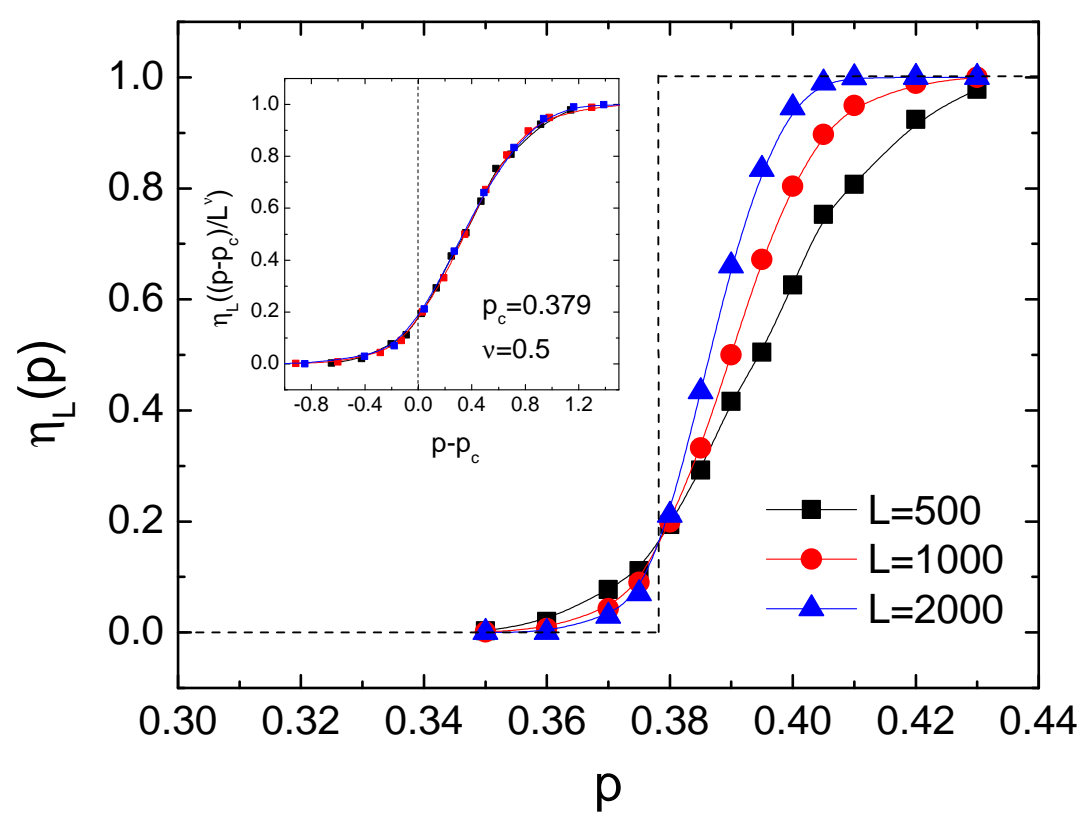

Figure 2. Main figure: The fraction of perfect matchings $\eta_{L}(p)$ as a function of the density $p$ of ones in the contact matrix $A$ for different lengths. The dashed line corresponds to the phase transition in the thermodynamic limit $L \rightarrow \infty$ at the critical point $p_{c}=0.379$. Inset: Finite-size scaling analysis of curves, corresponding to different lengths $L$. The fitting procedure yields $\nu \approx 0.5$ as the value of the transition width exponent. Each data point is averaged over $10^{4}$ instances.

\section{Improved analytical estimation of $p_{c}$ via arcs expansion: first order}

In this section, we show how to obtain a refined estimation of the perfect-imperfect transition point, using the formulation of the problem in terms of Dyck paths and combining exact combinatorial and mean-field techniques. The method is based on the observation that the arcs with smaller lengths are more likely to appear in the complete matching structure than those with higher lengths. Indeed, recall that locally, in the complete matching configuration, the arc opened at $i$ and closed at $j$ corresponds to the part of a Dyck path, starting by an "up"-step $\nearrow$ in position $i$ and ending by the first "down"-step \ at the same height in position $j$, cf. Fig. 1(b). Hence, this random walk between $i$ and $j$ is a Dyck path itself, and the probability to find an arc connecting $i$ and $j$, reads

$$
P(i, j)=\frac{C_{(k-1) / 2}}{2^{k+1}}
$$


Topological transition in disordered planar matching: combinatorial arcs expansion 8

where $k=j-i$; the nominator represents the total number of Dyck paths of length $k$, given by (2), and the denominator is the total number of possible random walks of this length.

From (7) we see that short links play an exceptional role in the formation of planar configurations: $P(i, j)$ is non-zero for odd $k$ only, and few first values are: $P(i, i+1)=1 / 4, P(i, i+3)=1 / 16, P(i, i+5)=1 / 32$, etc. In particular, in the large $L$ limit, about a half of all $L / 2$ arcs are the shortest ones (" $S$-arc") of length two (corresponding to red peaks in the Fig. 1(b)).

In our previous work [14 we have used this fact to provide an estimate for the perfect-imperfect transition point by considering the following approximation:

$$
\xi^{L / 2}(p)=\underbrace{p^{L / 4}}_{\text {long arcs }} \underbrace{\mathcal{P}_{S}^{(1)}(p)}_{\text {S-arcs }}
$$

that is, explicitly accounting for the correlations coming from shortest arcs, and assuming that all longer arcs give a mean-field contribution $p^{L / 4}$. Thus, the problem is reduced to placing $L / 4$ shortest arcs on the line of $L$ points, representing positions $(i, i+1)$, each position being allowed or forbidden as dictated by the contact matrix values $A_{i, i+1}$. Note that since the arcs can not share the same node, the $S$-arcs can not occupy neighboring positions $(i, i+1)$ and $(i+1, i+2)$ in such a placement.

We assume here that short arcs are uncorrelated apart from the non-overlap constraints. In real arc structures it is not the case: indeed, the total number of available structures with exactly $k$ shortest arcs in the absence of disorder is known to be given by the so-called Narayana number $N(2 L, k)$ [19, 20] instead of $C_{3 L / 4}^{L / 4}$ (see the computation below). However, correlations between short arcs are induced by the positions of longer ones, so assuming them to be uncorrelated seems to be a natural first approximation, while the correlations will arise naturally as one takes into account arcs of length 3, 5, etc. (see section 3 for more details).

We can express $\mathcal{P}_{S}^{(1)}(p)$ as follows:

$$
\mathcal{P}_{S}^{(1)}(p)=\frac{B_{S}^{(1)}(p)}{B_{S}^{(1)}(1)}
$$

where $B_{S}^{(1)}(p)$ is the number of ways to put uniformly $L / 4 S$-arcs, allowed by the contact matrix $A$ of density $p$, on a line of $L$ points, according to the non-touching constraint. It is easy to see that in the fully-connected case $p=1$ (all the positions 
Topological transition in disordered planar matching: combinatorial arcs expansion 9 are allowed), $B_{S}^{(1)}(1)=C_{3 L / 4}^{L / 4}$, corresponding to the placement of $L / 4$ objects among $L / 4 S$-arcs (that we will denote by $\square$ ) and $L / 2$ unmatched vertices (denoted by

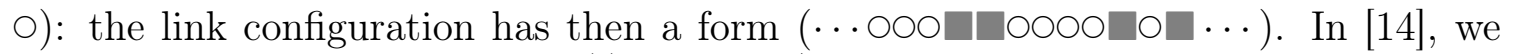

have used the approximation $B_{S}^{(1)}(p)=C_{3 p L / 4}^{L / 4}$, assuming that each position in the "circle-and-square" representation is allowed with probability $p$. Strictly speaking, it is not true, and gives only an upper-bound on the value of critical point computed at this level. Indeed, the density of "1" on the diagonal $A_{i, i+1}$ is equal to $p$ in the thermodynamic limit, but the "1" are distributed independently, meaning that they may correspond to incompatible neighboring positions $(i, i+1)$ and $(i+1, i+2)$; at the same time, the "circle-and-square" representation automatically incorporates the non-touching constraint. In this section we derive a contribution of the $S$-arcs (or peaks in the Dyck path representation, see Fig. 11), via an exact procedure.

\subsection{Formulation of the problem as a spin chain model}

The problem of placing $L / 4 S$-arcs on a line of $L$ a priori available positions $(i, i+1)$ can be equivalently formulated as a diluted Ising spin chain model. To each couple $(i, i+1)$ we associate a variable $\sigma_{i}$, equal to one if the arc is placed at this position, and and to zero otherwise. Because of the non-touching constraint, the product $\sigma_{i} \sigma_{i+1}$ must be always equal to zero. Moreover, we cannot count an arc as placed if the corresponding position is forbidden by the contact matrix, i.e. if $q_{i} \equiv A_{i, i+1}=0$. In what follows, we will denote allowed position $\left(q_{i}=1\right)$ by a square with a dot $(i, i+1) \equiv \sqcup$, and the forbidden position $\left(q_{i}=0\right)$ by an empty square $(i, i+1) \equiv \square$. The number of placements verifying these conditions are counted using the partition function

$$
Z=\sum_{\left\{\sigma_{i}\right\}} e^{-\beta H[q, \sigma]}
$$

in the limit $\beta \rightarrow \infty$, where $H[q, \sigma]$ is given by

$$
H[q, \sigma]=\sum_{i=1}^{L} q_{i} q_{i+1} \sigma_{i} \sigma_{i+1}
$$

with a counting constraint

$$
\sum_{i=1}^{L} q_{i} \sigma_{i}=\frac{L}{4} \equiv M
$$


Topological transition in disordered planar matching: combinatorial arcs expansion 10 Therefore, $Z$ can be expressed as

$$
Z=\sum_{\left\{\sigma_{i}\right\}} e^{-\beta \sum_{i=1}^{L} q_{i} q_{i+1} \sigma_{i} \sigma_{i+1}} \frac{1}{2 \pi i} \oint \mu^{\sum_{i=1}^{L} q_{i} \sigma_{i}-(M+1)} d \mu=\frac{1}{2 \pi i} \oint \mu^{-(M+1)} Z_{\mu} d \mu .
$$

Under periodic boundary conditions, $Z_{\mu}$ can be computed via the transfer matrix method:

$$
Z_{\mu}=\sum_{\left\{\sigma_{i}\right\}} e^{-\beta \sum_{i=1}^{L} q_{i} q_{i+1} \sigma_{i} \sigma_{i+1}+\frac{1}{2} \log \mu \sum_{i=1}^{L}\left(q_{i} \sigma_{i}+q_{i+1} \sigma_{i+1}\right)}=\operatorname{Tr} \prod_{i=1}^{L} T_{i, i+1},
$$

where the transfer matrix $T$ reads

$$
T_{i, i+1}=\left(\begin{array}{cc}
e^{-\beta q_{i} q_{i+1}} \mu^{\left(q_{i}+q_{i+1}\right) / 2} & \mu^{q_{i+1} / 2} \\
\mu^{q_{i} / 2} & 1
\end{array}\right)
$$

The solution is easy to obtain explicitly in the fully-connected case $p=1$, when $q_{i}=1$ for all $i$, and the chain has a form $(\square \odot \cdots \odot)$. In this case, we have (in the $\operatorname{limit} \beta \rightarrow \infty)$

$$
Z_{\mu}=\lambda_{1}^{L}+\lambda_{2}^{L},
$$

where $\lambda_{1,2}=(1 \pm \sqrt{1+4 \mu}) / 2$ are the eigenvalues of the matrix (15). We get

$$
Z_{\mu}=\frac{2}{2^{L}} \sum_{k=0}^{L / 2} C_{L}^{2 k}(1+4 \mu)^{k}=\frac{1}{2^{L-1}} \sum_{k=0}^{L / 2} \sum_{l=0}^{k} C_{L}^{2 k} C_{k}^{l}(4 \mu)^{l} .
$$

From (13), $Z=Z[M, L]$ is non-zero only for $l=M$, and finally we get (using combinatorial summation formula [21])

$$
Z[M, L]=\frac{1}{2^{L-1}} \sum_{k=0}^{L / 2} C_{L}^{2 k} C_{k}^{M}(2)^{2 M}=\frac{L}{M} C_{L-M-1}^{M-1} .
$$

In general, $p \neq 1$ case, some of the $L$ possible positions for the placement of the length-2 arcs are forbidden by the contact matrix. In other words, the contact matrix partitions the length- $L$ chain of all possible shortest arcs positions into pieces, representing the sequences of allowed positions, surrounded by forbidden ones: $(\cdots \square \vee \square \square \square \square \square \vee \square \square \cdots)$. Moreover, we see that the sequence of forbidden positions of arbitrary length is equivalent to a single forbidden position in the 
Topological transition in disordered planar matching: combinatorial arcs expansion11

product (14) up to a normalization constant, (.$\square \square \square \square \square \square \square \square \square \square \cdots) \Rightarrow$ $(\cdots \square \vee \bullet \square \square \square \square \bullet \square \cdots)$ : in this case the transfer matrix (15) reduces to

$$
T_{i, i+1}^{0}=\left(\begin{array}{ll}
1 & 1 \\
1 & 1
\end{array}\right)
$$

and we have $\left(T^{0}\right)^{2}=2 T^{0}$. Let us denote $q_{k}$ the density (in the large $L$ limit) of sequences of allowed positions of length $k: \square \underbrace{\ominus \cdots \boxminus \square}_{k}$. We have

$$
q_{k}=p^{k}(1-p)^{2}
$$

where the two factors $(1-p)$ come from the forbidden positions nearest to the first and to the last positions of the sequence, and each factor $p$ is the probability of an allowed position. It is easy to check that the overall density of allowed positions for the shortest arcs must be equal to $p$ :

$$
\sum_{k} k q_{k}=p(1-p)^{2}\left(1+2 p+3 p^{2}+\ldots\right)=p .
$$

Giving physical meaning to $B_{S}^{(1)}(p)$, we need to solve the following constrained independent set (CIS) problem: count the number of ways to distribute $L / 4$ arcs such that they do not touch each other, in the ensemble of allowed partitions. For each sequence it means that if a certain position is chosen, other arcs cannot be placed in the neighboring positions, even if these last are allowed by the contact matrix $A$. Note, however, that this global CIS problem is reduced to a set of local ones on the sequences with densities $q_{k}$ : since they are separated by at least one forbidden position, the distribution of $S$-arcs happens independently on each sequence.

\subsection{Strict bound}

Let us first ask a simpler question: what is the maximum number of arcs that can be placed, given the densities $q_{k}$ ? It is easy to see that for a piece of length $k$, at most $[(k+1) / 2] \equiv r_{k}$ positions can be occupied under non-touching constraint. Therefore, the maximum fraction of shortest arcs is

$$
\sum_{k}\left[\frac{k+1}{2}\right] q_{k}=p(1+p)(1-p)^{2}\left(1+2 p^{2}+3 p^{4}+4 p^{6}+\ldots\right)=\frac{p}{1+p} .
$$


Topological transition in disordered planar matching: combinatorial arcs expansion 12

As a by-product, we get a non-trivial strict bound on the value of $p_{c}$ : since we need to place at least $L / 4$ arcs, we immediately conclude that $p>1 / 3$. It coincides with the lower bound for RNA-type matching, found in [22] using the explicit construction in terms of integer-valued alphabets.

\subsection{Solution of the CIS problem}

Now let us return to the solution of the local CIS problem as it has been stated previously. Let us call $R_{m, k}$ the number of ways to put $m S$-arcs on the allowed sequence of positions of length $k$. For deriving this quantity, it is sufficient to notice that when one arc is placed, it is no longer possible to place another arc on a neighboring position due to the non-touching constraint. Hence, starting to put the $S$-arcs (represented by $\square$ ) one by one on the sequence of length $k$, we should forbid the position next to the placement position (this constrained position will be denoted by $\otimes)$ :

$$
\underbrace{\ominus \square \boxminus \square \square \otimes \cdots}_{k} .
$$

In other words, we have to count the number of ways to put $m$ objects $(\square)$ on a sequence of length $k$. This number is given by $C_{k-2 m+m}^{m}$ if the last position of the sequence is left free $(\square)$, and to $C_{(k-1)-2(m-1)+(m-1)}^{m-1}$, if it is occupied $(\square)$ :

$$
R_{m, k}=C_{k-m}^{m}+C_{k-m}^{m-1}=C_{k-m+1}^{m} .
$$

This result is in perfect agreement with the expression for the fully-connected case, when all the $L$ positions are allowed: $B_{S}^{(1)}(1)=R_{L / 4, L}=C_{3 L / 4}^{L / 4}$. Notice that (24) is different from the formula (18) (with obvious correspondences $M \rightarrow m$ and $L \rightarrow k$ ), because the last one, being derived with periodic boundary conditions, corresponds to a ring of allowed positions, rather than to a sequence. Anyway, one of these expressions can be easily derived from another by noticing that a CIS problem on the sequence is equivalent to a CIS problem on a ring with one additional forbidden position, leading to the equality

$$
R_{m, k}+R_{m-1, k-2}=Z[m, k+1],
$$

which is verified, given (18) and (24).

Given the solution of the local CIS problem (24), it is easy to construct the solution of the global problem. Let us introduce a generating function for a piece of 
Topological transition in disordered planar matching: combinatorial arcs expansion 13

length $k$ :

$$
Q_{k}(s)=\sum_{m=0}^{r_{k}} R_{m, k} s^{m}
$$

Hence the generating function for the whole chain of $L$ a priori available positions for the $S$-arcs reads

$$
Q(s)=\prod_{k=1}^{L}\left(Q_{k}(s)\right)^{L q_{k}},
$$

or, explicitly,

$$
Q(s)=(1+s)^{L p(1-p)^{2}}(1+2 s)^{L p^{2}(1-p)^{2}}\left(1+3 s+s^{2}\right)^{L p^{3}(1-p)^{2}}\left(1+4 s+3 s^{2}\right)^{L p^{4}(1-p)^{2}} \ldots
$$

Since we want to place $L / 4$ shortest arcs, we are interested in the coefficient behind the $s^{L / 4}$ : this is exactly the quantity $B_{S}^{(1)}(p)$. This coefficient is given by the integration of $Q(s) / s^{L / 4}$ around zero:

$$
B_{S}^{(1)}(p)=\frac{1}{2 \pi i} \oint d s \exp \left[L\left((1-p)^{2} f_{s}(p)-1 / 4 \log s\right)\right],
$$

where

$$
f_{s}(p)=\sum_{k=1}^{L} p^{k} \log \left(\sum_{m=0}^{[(k+1) / 2]} C_{k-m+1}^{m} s^{m}\right) .
$$

Using explicit summation [21, this result can be written as

$$
f_{s}(p)=\sum_{k=1}^{L} p^{k} \log \left(\frac{(1+\sqrt{1+4 s})^{k+2}-(1-\sqrt{1+4 s})^{k+2}}{2^{k+2} \sqrt{1+4 s}}\right) .
$$

Each term in this sum is decreasing, so in numerical calculations we can approximate this function by partial sums to some order $k_{0}$. The integral (29) can be treated by the steepest descent method. The saddle-point equation reads

$$
(1-p)^{2} \frac{\partial f_{s}(p)}{\partial s}=\frac{1}{4 s} .
$$

Given the solution $s^{*}$ of the equation (32), one gets the expression for $B_{S}^{(1)}(p)$ :

$$
B_{S}^{(1)}(p)=\exp \left[L\left((1-p)^{2} f_{s^{*}}(p)-1 / 4 \log s^{*}\right)\right] .
$$

Approximating the large deviation function (30) by partial sums up to the fifteenth order, and combining with (9), (8) and (5), we get a fast convergence to the prediction of the critical point $p_{c}^{*}=0.3376$, see Fig. 3, providing an expected shift to a lower value from the result $p_{c}^{*}=0.35$, originally found at this level of expansion in [14]. 
Topological transition in disordered planar matching: combinatorial arcs expansion14

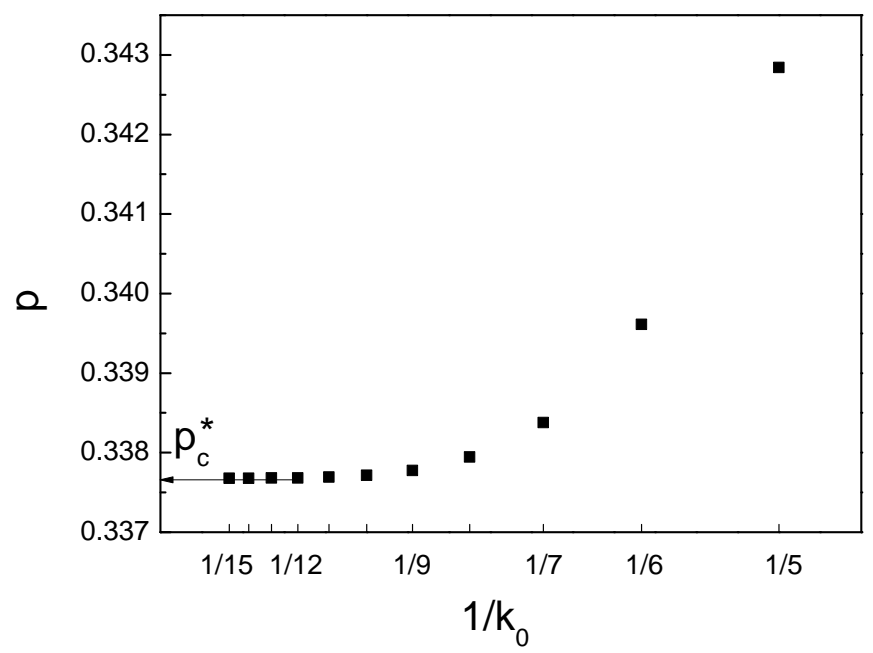

Figure 3. Estimation of the prediction $p_{c}^{*}$ for the critical value $p_{c}$ at the first order of arcs expansion. Each point represents a corresponding estimation when $\sqrt{30}$ is approximated by a partial sum up to the lengths $k_{0}$. The estimations demonstrate a fast convergence with $k_{0}$ to the value $p_{c}^{*}=0.3376$.

\section{Improved analytical estimation of $p_{c}$ via arcs expansion: beyond the first order}

The estimation obtained in the previous section can be systematically improved by considering correlations arising form higher-length arcs. The idea is to use not only the information from the diagonal $A_{i, i+1}$ of the contact matrix, but also from the diagonal $A_{i, i+3}$, i.e. to take into account the constraints on the placement of $S$-arcs (shortest, length-2 arcs), that come from the placement of the length-4, or next-toshortest $(N S)$ arcs. Therefore, we can write, as previously

$$
\xi^{L / 2}(p)=\underbrace{p^{L / 4}}_{\text {longer arcs }} \underbrace{\mathcal{P}_{S}^{(2)}(p)}_{\text {S-arcs }},
$$

but now the influence of the $L / 16 N S$-arcs is accounted in the factor $\mathcal{P}_{S}^{(2)}(p)$ representing $L / 4 S$-arcs. As before, we will compute the contributions of the shortest arcs under the correlations arising from the placement of the $N S$-arcs, and treat the contribution arising from the longer arcs in a mean-field manner.

In other words, the problem is now reduced to the placement of both $S$ and $N S$ arcs that respect the constraints imposed by the contact matrix $A$. Obviously, 
the placement of arcs of one type introduces additional constraints on the placement of those of other type. First, some of the places will become forbidden because of the non-crossing constraints. Second, if we are interested in the complete matching configurations, placing a length- 4 arc automatically means placing a length-2 arc underneath. Therefore, our goal is to place altogether $L / 16 N S$-arcs, each covering an $S$-arc (in what follows, we will denote a placement of such a construction by $\mathbf{\square}$ ), $L / 4-L / 16=3 L / 16$ remaining $S$-arcs (as usual, they will be denoted by $\square$ ) and $L-4 \times L / 16-2 \times 3 L / 16=3 L / 8$ unmatched vertices. The placements are subject to the non-touching constraints. Proceeding in the same way as in the previous section, we can write

$$
\mathcal{P}_{S}^{(2)}(p)=\frac{B_{S}^{(2)}(p)}{B_{S}^{(2)}(1)},
$$

where the factor $B_{S}^{(2)}(p)$ represents the contributions of the $S$-arcs under the correlations arising from the presence of the $N S$-arcs. The denominator of the product (35) is given by a multinomial coefficient

$$
B_{S}^{(2)}(1)=\frac{\frac{5 L}{8} !}{\frac{L}{16} ! \frac{3 L}{16} ! \frac{3 L}{8} !}=C_{5 L / 8}^{L / 16} C_{9 L / 16}^{3 L / 16} .
$$

The multinomial coefficient has the following physical sense: it counts the number of ways to place length- 4 constructions, length- 2 arcs and unmatched vertices when all the places are available by the contact matrix, i.e. to count the number of link configurations of the form $(\cdots \circ \circ)$ binomial coefficients, describing the following placements: first put $L / 16$ length- 4 constructions among $L / 16+3 L / 16+3 L / 8=5 L / 8$ objects, and then put $3 L / 16$ remaining $S$-arcs among $3 L / 16+3 L / 8=9 L / 16$ available places. In what follows, we will evaluate the numerator of 35 .

\subsection{Localization of the problem}

The computation of $B_{S}^{(2)}(p)$ requires counting the number of placements of $S$ and $N S$ arcs allowed by the contact matrix and respect the non-touching constraint. In order to facilitate the manipulations, we shall use the following convention: we say that the length-4 construction (composed of a $N S$-arc which cover $S$-arc underneath) is placed at position $i$ if $i$ is the starting point of the corresponding $S$-arc, and the whole construction occupies positions $i-1, i, i+1, i+2$. For simplicity, when discussing the 
placement of $N S$ arcs, we will also assume that the corresponding $S$-arc is always placed below. The probability that the $N S$ arc can be placed at position $i$ is hence given by $p(1-(1-p))=p^{2}$; if the position $i$ is indeed allowed, it will be denoted by $\boxplus$. Of course, a $S$-arc only can also be placed at position $\boxplus$. We will mark by $\square$ the positions at which a $S$-arc can be placed, but not the $N S$-arc (it happens with probability $p(1-p))$. As previously, a position at which none of the arcs can be placed will be marked as $\square$.

We proceed in a manner similar to what has been done in the section 2 . Again, we notice that the global placement problem on a string of the form $(\cdots \square \square \boxplus \square \square \square \boxplus \square \square \square \cdots)$ can be reduced to a set of local CIS problems on independent pieces, separated by a forbidden position $\square$. These sequences have to be of a special form so that the choice of the placement on one piece doesn't interfere with the placements on the neighboring pieces.

The optimal form of each independent piece can be shown to be

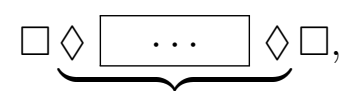

where $\diamond$ represents a position on which a $N S$-arc is not allowed, i.e. either $\square$ or $\square$. This requirement is based on the observation that two $N S$-arcs placed in neighboring sequences have to be separated by at least three non-allowed positions in order to avoid interference due to the non-touching of the arcs. Same restrictions occur also in the boxed part of the sequence. One sees that forbidden positions $\square$ must have at least one neighboring position $\boxplus$ that allow for the placement of the $N S$-arcs; otherwise, the sequence in question can be separated in two independent pieces according to the definition above.

Each independent piece of length $k$ can be represented by a certain number of different sequences that satisfy the restrictions below. We illustrate these possible variants in the Table 1 for different lengths up to $k=3$. The probability of each sequence is fully determined through the number of positions of different sorts: $k^{\prime}$ of $\boxplus, k^{\prime \prime}$ of $\square$ and $k-k^{\prime}-k^{\prime \prime}$ of $\square$. The density of each independent sequence is then given by

$$
u_{k^{\prime}, k^{\prime \prime}, k}=p^{2 k^{\prime}}(p(1-p))^{k^{\prime \prime}}(1-p)^{k-k^{\prime}-k^{\prime \prime}}(1-p)^{2}\left(1-p^{2}\right)^{2},
$$

where the factor $(1-p)^{2}$ comes from the two forbidden positions $\square$ at the endings of the sequence, and the factor $\left(1-p^{2}\right)^{2}$ ensures that the next-to-forbidden position doesn't allow for the placement of a $N S$-arc, i.e. is $\diamond$. 
Topological transition in disordered planar matching: combinatorial arcs expansion 17

\begin{tabular}{|c|c|c|c|c|}
\hline$k$ & $\alpha$ & Representation & $u_{k^{\prime}, k^{\prime \prime}, k} /\left((1-p)^{2}\left(1-p^{2}\right)^{2}\right)$ & Non-zero $Y_{m, n, k^{\prime}, k^{\prime \prime}}^{\alpha, k}$ \\
\hline 1 & 1 & $\square \underbrace{\square} \square$ & $p(1-p)$ & $Y_{0,1,0,1}^{1,1}=1$ \\
\hline 2 & 1 & $\square \underbrace{\dot{\ominus} \cdot \dot{\theta}} \square$ & $(p(1-p))^{2}$ & $Y_{0,1,0,2}^{1,2}=2$ \\
\hline 3 & 1 & $\square \underbrace{\ominus \dot{\theta} \cdot \square}$ & $(p(1-p))^{3}$ & $Y_{0,1,0,3}^{1,3}=3, Y_{0,2,0,3}^{1,3}=1$ \\
\hline 3 & 2 & $\square \underbrace{\ominus \oplus \boxminus \square}$ & $p^{2}(p(1-p))^{2}$ & $Y_{0,1,1,2}^{2,3}=2, Y_{0,2,1,2}^{2,3}=Y_{1,0,1,2}^{2,3}=1$ \\
\hline 3 & 3 & $\square \underbrace{\ominus \boxplus \square} \square$ & $p^{2}(p(1-p))(1-p)$ & $Y_{0,1,1,1}^{3,3}=Y_{1,0,1,1}^{3,3}=1$ \\
\hline 3 & 4 & $\square \underbrace{\square \boxplus \square} \square$ & $p^{2}(p(1-p))(1-p)$ & $Y_{0,1,1,1}^{4,3}=Y_{1,0,1,1}^{4,3}=1$ \\
\hline 3 & 5 & $\square \underbrace{\square \boxplus \square}$ & $p^{2}(1-p)^{2}$ & $Y_{1,0,1,0}^{5,3}=1$ \\
\hline 4 & 1 & 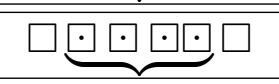 & $\overline{(p(1-p))^{4}}$ & $Y_{0,1,0,4}^{1,4}=4, Y_{0,2,0,4}^{1,4}=3$ \\
\hline 4 & & $\cdots$ & & \\
\hline
\end{tabular}

Table 1. The explicit representation of different possible elementary sequences for lengths up to $k=3$. The local CIS problem is solved independently on each sequence, providing the weights $Y_{m, n, k^{\prime}, k^{\prime \prime}}^{\alpha, k}$.

\subsection{Solution of the CIS problem}

As it has been done in the section 2, we can try to solve the local CIS problem on these independent sequences. However in the present case the combinatorics is rather involved since the solution inside each block depends on the distribution of forbidden and allowed positions. Nevertheless, if we truncate the series at some length $k_{0}$ (as it has been done at first order of arcs expansion), these solutions can be computed for each sequence via explicit enumeration, for the pseudo-code see Table 2, Let us call $Y_{m, n, k^{\prime}, k^{\prime \prime}}^{\alpha, k}$ a number of ways to put $m N S$-arcs (hiding corresponding $S$ arcs) and $n$ uncovered $S$-arcs on the sequence of length $k$ comprising $k^{\prime}$ positions $\boxplus$ and $k^{\prime \prime}$ positions $\square$, where $\alpha$ counts the number of different sequences having the same density $u_{k^{\prime}, k^{\prime \prime}, k}$.

Then, again, a generating function for a piece $\left(k^{\prime}, k^{\prime \prime}, k\right)$ can be introduced:

$$
W_{k^{\prime}, k^{\prime \prime}, k}^{\alpha}(s)=\sum_{m} \sum_{n} Y_{m, n, k^{\prime}, k^{\prime \prime}}^{\alpha, k} s^{m+n}
$$

where the power of $s$ counts the overall number of $S$-arcs placed at each individual sequence. Hence the generating function for the whole chain of $L$ a priori available 
set $k_{0} ;$
set all $Y_{m, n, k^{\prime}, k^{\prime \prime}}^{\alpha, k}=0$
for $k=1, \ldots, k_{0}$
$\quad$ for $\alpha=1, \ldots, \alpha_{\max }(k)$

Generate correct configuration $\alpha$ with $k^{\prime} \boxplus, k^{\prime \prime} \square$ and $\left(k-k^{\prime}-k^{\prime \prime}\right) \square$ :

Choose and distribute $k^{\prime} \boxplus$;

Neighbors of $\boxplus$ are $\diamond$, i.e. either $\square$ or $\square$;

All other elements are $\square$;

for $m=0, \ldots, k^{\prime}$

for $n=0, \ldots, k^{\prime}+k^{\prime \prime}$

Try to place $m N S$-arcs $\square$ and $n S$-arcs $\square$ on allowed positions;

if non-touching constraints satisfied: increment $Y_{m, n, k^{\prime}, k^{\prime \prime}}^{\alpha, k}$;

end

end

end

end

return $Y_{m, n, k^{\prime}, k^{\prime \prime}}^{\alpha, k}$

Table 2. Counting algorithm for the computation of coefficients $Y_{m, n, k^{\prime}, k^{\prime \prime}}^{\alpha, k}$ up to a maximum sequence length $k_{0}$.

positions reads

$$
W(s)=\prod_{k=1}^{k_{0}} \prod_{\left(k^{\prime}, k^{\prime \prime}\right)}\left(\prod_{\alpha} W_{k^{\prime}, k^{\prime \prime}, k}^{\alpha}(s)\right)^{L u_{k^{\prime}, k^{\prime \prime}, k}} .
$$

We want to control that the total number of $S$-arcs is $L / 4$, so we are interested in the coefficient behind the $s^{L / 4}$ : this will give us precisely the quantity $B_{S}^{(2)}(p)$. This coefficient is obtained by the integration of $W(s) / s^{L / 4}$ around zero:

$$
B_{S}^{(2)}(p)=\frac{1}{2 \pi i} \oint d s \exp \left[L\left(g_{s}(p)-1 / 4 \log s\right)\right]
$$

where $g_{s}(p)=\log W(s) / L$. The saddle-point equation is

$$
\frac{\partial g_{s}(p)}{\partial s}=\frac{1}{4 s} \text {. }
$$

Given the solution of the saddle-point equation $s^{*}$, we have

$$
B_{S}^{(2)}(p)=\exp \left[L\left(g_{s^{*}}(p)-1 / 4 \log s^{*}\right)\right] .
$$


Combining (35), (34) and (5), and going in maximum length up to $k_{0}=15$, we get a fast convergence to the value $p_{c}=0.3743$ which is very close to the value found in numerical simulation, see Fig. 4 .

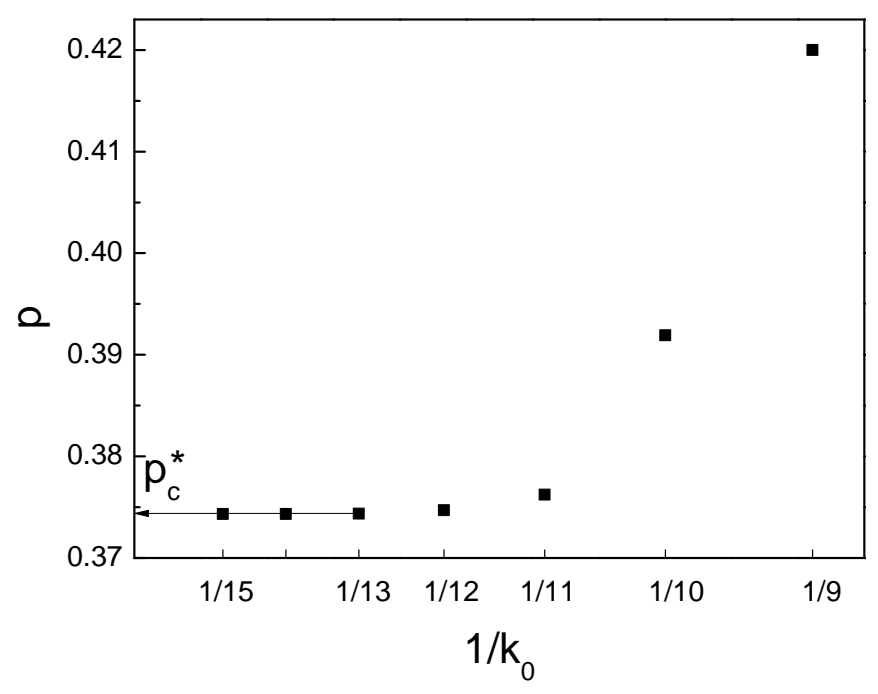

Figure 4. Estimation of the prediction $p_{c}^{*}$ for the critical value $p_{c}$ at the second order of arcs expansion. Each point represents a corresponding estimation when (40) is approximated by a partial product up to the lengths $k_{0}$. The estimations demonstrate a fast convergence with $k_{0}$ to the value $p_{c}^{*}=0.3743$.

\subsection{Next orders}

In principle, this estimation can be improved further by considering the contributions from higher order arcs. For example, for the next order, including length-6, or nextto-next-to-shortest $(N N S)$ arcs, we can write

$$
\xi^{L / 2}(p)=\underbrace{p^{L / 4}}_{\text {longer arcs }} \underbrace{\mathcal{P}_{S}^{(3)}(p)}_{\text {S-arcs }},,
$$

where now the $S$-arcs are placed according to the restrictions imposed by $L / 32$ $N N S$-arcs and $L / 16 N S$-arcs. A subtlety here is that if we are interested in the fully-matched configurations, each of the $N N S$-arcs may hide either $2 S$-arcs, either a nested structure of $N S$ and $S$ arcs. Hence, placing $L / 32 N N S$-arc on a certain position (allowed with the probability $p\left(1-\left(1-p^{2}\right)^{2}\right)$ ) automatically means placing 
Topological transition in disordered planar matching: combinatorial arcs expansion 20

$L / 64 N S$-arcs and $3 L / 64 S$-arcs; at the same time, $L / 16-L / 64=3 L / 64$ of $N S$-arcs are still remaining outside the placed $N N S$-arcs, and $L / 4-3 L / 64-3 L / 64=5 L / 32$ of $S$-arcs are still remaining outside both $N N S$ and $N S$. We have to place them altogether with $5 L / 16$ unmatched vertices. Therefore, if we write, as usual,

$$
\mathcal{P}_{S}^{(3)}(p)=\frac{B_{S}^{(3)}(p)}{B_{S}^{(3)}(1)},
$$

the factor $B_{S}^{(3)}(1)$ will be given by

$$
B_{S}^{(3)}(1)=\frac{\frac{35 L}{64} !}{\frac{L}{32} ! \frac{1 L}{64} ! \frac{5 L}{32} ! \frac{5 L}{16} !}=C_{35 L / 64}^{L / 32} C_{33 L / 64}^{3 L / 64} C_{15 L / 32}^{5 L / 32} .
$$

The calculation of the factor $B_{S}^{(3)}(p)$ would involve, as in the previous sections, the partitioning of the problem into a set of local CIS problems. The solution to the global problem could then be obtained by imposing that overall number of $S$-arcs is fixed to $L / 4$. Note that, in principle, we could have thought about fixing the number of longer arcs as well: the number of $N S$-arcs to $L / 16$, the number of $N N S$-arcs to $L / 32$, etc. This method would require to introduce several counting variables in the generating function; then we would need to perform the saddle-point analysis in a multi-dimensional space, which may lead to possible numerical instabilities. At the same time, the idea to control the number of $S$-arcs, subject to constraints due to the presence of longer arcs, leads to a saddle-point equation with respect to one variable only at each order of the expansion, which is easier to control.

\section{Beyond Bernoulli model of planar matching for non-integer alphabets}

The planar matching problem has an application to a toy formulation of the optimal secondary structure problem in RNA molecules. A real RNA is a single-stranded polymer composed of four types of nucleotides (A, C, G and U). The secondary structure of RNA is represented by the chemical bonds between the stable WatsonCrick pairs A-U and G-C in the folded state. The simplest theories designed for the study of statistical properties of the RNA secondary structures usually focus on the random RNAs in which the nucleotide sequence is random, and assume the saturation of base pairings and the exclusion of the pseudoknots which are known to be rare in real RNAs [9]. These assumptions imply that the secondary structure 
can be represented as a planar diagram, the solution of the planar matching problem considered in this paper, where the contact matrix $A$ encodes the disorder in the primary RNA sequence of nucleotides [14, 8]. In this picture, the parameter $p$ of the Bernoulli contact matrix $A$ is in the one-to-one correspondence with the number of nucleotides, or alphabet, $c$ (equal to four for real RNAs) in the primary sequence: since $p$ characterizes the average density of contacts that each base may have, we simply have $p=1 / c$. The contact matrix representation of the sequence disorder is a convenient tool since it allows to expand the study over the non-integer alphabets.

Although it is clear that, given the pairing complementarity rules, one can always build a contact matrix from a given primary sequence, the opposite in general is not true. Indeed, in the Bernoulli model, each element of the matrix is generated independently according to the probability distribution (1), hence, it lacks the transitivity: even if the elements $A_{i j}$ and $A_{j k}$ appear to be equal to one in the contact matrix, the element $A_{i k}$ might be zero. However, this limitation is irrelevant in the thermodynamic limit, i.e. when the length of the sequence $L \rightarrow \infty$ [14.

Still, it would be interesting to understand whether there is a way to construct explicit random primary sequence that could model the primary sequences with non-integer alphabets. In the context of the phase transition described in the section 1. we have seen that there is a critical value $p_{c} \approx 0.379$ of the bond formation probability that separates the regions of optimal and non-optimal structures; this critical probability corresponds to the critical alphabet $c_{c r} \approx 2.64$ in this generalized primary sequence setting. In this section, we address the following questions: i) Is it possible to construct explicitly a random sequence with transitive or partially transitive matching rules that would correspond to a non-integer alphabet $c$, i.e. have a density $p=1 / c$ of ones in the contact matrix, generated according to this sequence? ii) Do these sequences exhibit an analogous critical behavior as the Bernoulli model with the same parameter $p$, and what is the relation to the behavior of the Bernoulli model?

\subsection{Construction of the non-integer alphabets}

For the models of random sequences, we consider a set of monomers of different types,

that we will call A, B, C, etc. Perhaps the most natural way to think of the noninteger alphabet $2<c<3$ is to consider three types of monomers: $\mathrm{A}, \mathrm{B}$ and $\mathrm{C}$, mixed 
together. For the sake of simplicity, we will assume that the links can be established between the monomers of the same type, A-A, B-B and C-C. It is clear that if three types of monomers are distributed randomly and independently along the sequence, this corresponds to an alphabet $c=3$. However, the effective non-integer alphabet $c<3$ can be modelled by assuming that the distribution of monomers along the chain is correlated. Suppose that starting from the first randomly chosen monomer, each next monomer in the sequence is generated according to the Markov-like process, with the probabilities that depend on the monomer at the previous step:

\begin{tabular}{c|ccc} 
& $\mathrm{A}$ & $\mathrm{B}$ & $\mathrm{C}$ \\
\hline $\mathrm{A}$ & $1-2 \epsilon$ & $\epsilon$ & $\epsilon$ \\
$\mathrm{B}$ & $\epsilon$ & $1-2 \epsilon$ & $\epsilon$ \\
$\mathrm{C}$ & $\epsilon$ & $\epsilon$ & $1-2 \epsilon$
\end{tabular}

This probability matrix is chosen to be symmetric with respect to all monomer types. Each monomer type appears in subsequences unless it is changed to another type: $(\cdots$ A A A B B B B A C C C ...). It has been proven in [22] that if the perfect matching solutions exist, there is at least one in which the neighboring monomers of the same type are matched together. It means that without any loss of generality, we can match the repeated monomers along the chain; this way, each subsequence of a certain type of even or odd length is reduced to one or zero monomers of this type, respectively: $(\cdots \mathrm{B}$ A A A C $\cdots) \rightarrow(\cdots$ B A C $\cdots)$.

The variation of the parameter $\epsilon$ from 0 to $1 / 3$ then gives a sequence that corresponds to an effective alphabet $c$ in a range from 1 to 3 . The relation between $\epsilon$ and $c$ can be estimated as follows:

$$
c=\left(\frac{1}{\epsilon}-2\right)^{2 \epsilon} \frac{1}{1-2 \epsilon} .
$$

The rational behind this estimation is based on the concept of Shannon information entropy [23]. The entropy rate of this markovian sequence is given by

$$
S=-\sum_{a=\mathrm{A}, \mathrm{B}, \mathrm{C}} P(a) \sum_{b=\mathrm{A}, \mathrm{B}, \mathrm{C}} P(b \mid a) \log P(b \mid a),
$$

where $P(a)=1 / 3$ is an a priori probability for the monomer of a certain type, and $P(b \mid a)$ is a conditional probability that the monomer of the type $a$ is followed by the monomer of the type $b$; this probability is given by the probability matrix of the 
considered Markov process. On the other hand, if one assumes that the sequences constructed in this way are described by an effective alphabet with $c$ equivalent monomers, we simple have

$$
S=-\sum_{a=1}^{c} \hat{P}(a) \log \hat{P}(a)
$$

with $\hat{P}(a)=1 / c$. The combination of (48) and (49) gives us the relation (47). Thus constructed alphabet will be referred to as the "correlated" alphabet.

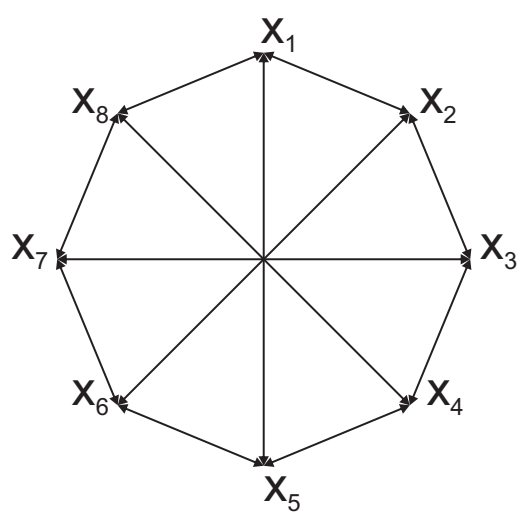

Figure 5. An example of the matching rules in the $(8,3)$ rational alphabet model. In this representation, a link between the monomers of types $X_{i}$ and $X_{j}$ means that they can potentially form a bond in the matching structure.

Another model that can be suggested for non-integer alphabets can be obtained using the observation that each non-integer alphabet $c$ can be approximated by a rational fraction $c=P / Q$. Imagine a random polymer with $P$ different monomer types $X_{1}, \ldots, X_{P}$, but now allow each of them to bind only with $Q$ other monomer types. The complementary rules can be depicted as a $P$-polygon with $Q-2$ additional links, where each link means a possible matching between two monomers, see Fig. 5 for an example with $P=8$ and $Q=3$. The "commutation relations" for the 
monomers read

$$
\begin{aligned}
& \left\{X_{i}, X_{i \pm j}\right\}=1 \text { for } 1 \leq j \leq[Q / 2] \\
& \left\{X_{i}, X_{i}\right\}=1 \text { if } Q \text { and } P \text { odd } \\
& \left\{X_{i}, X_{i+P / 2}\right\}=1 \text { if } Q \text { odd and } P \text { even, } \\
& \left\{X_{i}, X_{i+j}\right\}=0 \text { otherwise }
\end{aligned}
$$

where $\left\{X_{i}, X_{k}\right\}$ represents a presence (one) or absence (zero) of possible matching between the two monomers $X_{i}, X_{k}$; the periodic condition $X_{i+P} \equiv X_{i}$ is understood. We will call this model a $(P, Q)$ "rational alphabet". Note that by construction this alphabet is non-transitive. A particularity of this model is that there is an infinite number of ways to represent $c$ as a fraction. Let us call $P^{*}$ and $Q^{*}$ as the minimal $P$ and $Q$ that give $c=P / Q$. Then $P=n P^{*}$ and $Q=n Q^{*}$ for an arbitrary integer $n$ give the same value of $c$, although involving a different number of monomer types. In the thermodynamic limit $L \rightarrow \infty$ it will make no difference since the density of ones in the contact matrix will be exactly $p=Q / P$, but for finite $L$ it may result in a different behaviors for the models with $c=P^{*} / Q^{*}, c=2 P^{*} / 2 Q^{*}$, etc. In order to minimize this effect, we place ourselves in the context of the urn model, in which the number of monomers of different sorts in the sequence are restricted to be equal.

\subsection{Perfect matching transition for non-integer alphabets}

We have investigated the behavior of both correlated and rational alphabets with respect to the perfect matching transition. To this purpose, we start by drawing random sequences corresponding to a particular $c$, then construct the contact matrix $A$ according to the matching rules defined in both models, and, finally solve the matching problem for each instance by the dynamical programming algorithm.

Surprisingly, we have not observed any transition with variation of $c$ for the model of the correlated alphabet. In fact, if $c>2$ (or $\epsilon>0.1135$ ) in this model, there is always a non-zero fraction of sequences that do not allow for the complete matching solutions. A possible reason for this is that due to the structure of the sequence, the matching on each subsequence is easy, but then the sequence is reduced to the primary structure of length $O(L)$ which corresponds effectively to the alphabet $c=3$, while we know, that for this alphabet (for $p=1 / 3$ ) in general there is no solution to the perfect matching problem. 
Topological transition in disordered planar matching: combinatorial arcs expansion 25

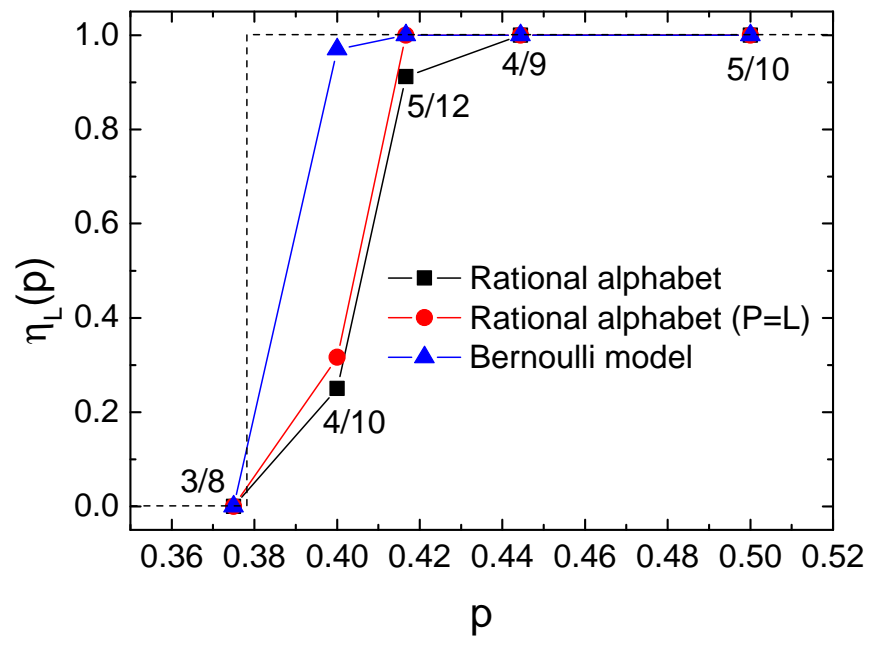

Figure 6. The fraction of perfect matchings $\eta_{L}(p)$ as a function of the density $p$ of possible contacts in the model of $(P, Q)$ rational alphabet (squares, the respective values of $p=P / Q$ are indicated on the plot), fluctuation-free Bernoulli model (rational alphabet model with $P=L$, circles) and Bernoulli model (triangles). The simulations have been performed for $L=2000$ and averaged over 10000 instances.

To the contrary, the rational alphabet model clearly exhibits a critical behavior in the matching problem. In the Fig. 6, we present the numerical results for the fraction, $\eta_{L}(p)$, of contact matrices that allow perfect matchings for different $p$. To avoid the sensitivity on the value of $P$ due to the finite size effects, we have chosen simple test values $p=Q / P$ with similar $P$ in the range $P \in[8,12]$. The number of perfect matching in these points are compared to the special case of the limit $P=L$, i.e. when all $L$ randomly distributed in the chain monomers are distinct, however being able to match $Q=p L$ other monomers in the chain. This limit corresponds to the fluctuation-free Bernoulli model, in which every line of the matrix $A$ contains exactly $p L$ of ones, without fluctuations of order $\sqrt{L}$ that appear in the model defined by (11). Rational alphabets give similar predictions, which are however very different with respect to the predictions of the Bernoulli model. This difference illustrates the "positive" role of fluctuations of the number of contacts in the Bernoulli matrix from the viewpoint of the matching problem. 
Topological transition in disordered planar matching: combinatorial arcs expansion 26

\section{Conclusion}

The statistical properties of the planar matching models considered in this paper are fully determined by only a few parameters: one for Bernoulli model $(p)$ and for the model with a correlated alphabet $(c$, or $\epsilon)$, or two for the rational alphabet model $(P$ and $Q)$. Nevertheless, these disordered models exhibit a non-trivial critical behavior. Although an instance of the matching problem can be solved by the dynamical programming algorithm with a polynomial complexity $\left(L^{3}\right.$, where $L$ is the size of an instance of the problem), the analytical estimation of the critical point is hard due to the quenched nature of the disorder.

In this paper, we have developed a combinatorial procedure that allows to obtain successive estimations for the value of the critical point in the previously studied Bernoulli model. This arcs expansion procedure benefits from the observation that the arcs of small length play an exceptional role in the complete matching structures. The key ingredient that makes the problem solvable is the fact that the global constraint satisfaction problem can be reduced to a set of local ones that are easier to solve. The developed method hence provides an insight into the fundamental structural properties of the fully-matched structures.

We have also considered a toy application in the context of random RNA-type sequences. We have designed two simple models that allow for a representation in terms of a finite set of monomer types and give a concrete sense to the notion of the effective non-integer alphabet. Although a simple model of a transitive correlated alphabet did not show a phase transition with a variation of the density of allowed contacts, the non-transitive rational alphabet clearly manifested the corresponding critical behavior. As a by-product, we have observed the positive influence of fluctuations in the Bernoulli model by studying the limit of a large number of monomer types that corresponds to the fluctuations-free case of the Bernoulli model.

Finally, let us emphasize that the models considered in this paper can not be regarded as relevant for the secondary structure formation in real RNAs. Indeed, real RNAs are characterized by varying energies of different Watson-Crick parings, limits on minimal lengths of stems (or the so-called stacking energies) and loops, presence of pseudoknots, etc. Therefore, the obtained exact quantitative results are certainly not directly applicable to a real RNA. However, as it has been argued in [13], the considered morphological transition is a universal phenomenon, persisting as well in more detailed models of RNA secondary structure formation: it represents 
Topological transition in disordered planar matching: combinatorial arcs expansion 27

a transition from a highly degenerate nearly-perfect structure for small nucleotide alphabets to the unique, but highly defective imperfect structure for large alphabets. We anticipate that the techniques developed in this paper will be useful for the analysis of the transition of this type in more general models.

\section{Acknowledgments}

The authors are thankful to C. Moore and V. Stadnichuk for fruitful conversations. This work is partially supported by "Investissements d'Avenir" LabEx PALM (ANR10-LABX-0039-PALM) project PRONET, and the IRSES projects FP7-PEOPLE2010-IRSES 269139 DCP-PhysBio and FP7-PEOPLE-2014-IRSES 612707 Dionicos. O.V.V, S.K.N. and M.V.T. are grateful to the Higher School of Economics program for basic research.

\section{References}

[1] Brézin, E, Itzykson, C, Parisi, G, \& Zuber, J. B. Communications in Mathematical Physics 59, 35-51 (1978).

[2] Abrikosov, A. A \& Gorkov, L. P. Methods of quantum field theory in statistical physics. (Courier Dover Publications) (1975).

[3] Saito, R. Journal of the Physics Society Japan 59, 482-491 (1990).

[4] Mehta, M. L. Random matrices. (Academic press) (2004).

[5] de Gennes, P. G. Biopolymers 6, 715-29 (1968).

[6] Nussinov, R \& Jacobsont, A. B. Proceedings of the National Academy of Sciences 77, 6309-6313 (1980).

[7] Müller M. Physical Review E 67021914 (2003).

[8] Bundschuh, R \& Hwa, T. Physical Review E 65, 031903 (2002).

[9] van Batenburg, F. H. D., Gultyaev, A. P., Pleij, C. W. A., Ng, J. \& Oliehoek, J. Nucl. Acids Res. 28 (1), 201-204 (2000).

[10] Lovász, L. \& Plummer, M. D. Matching theory (American Mathematical Society) (2009).

[11] Kasteleyn, P. W. Physica 27, 12, 1209-1225 (1961).

[12] Vernizzi, G, Orland, H, \& Zee, A. Physical Review Letters 94, 168103 (2005).

[13] Valba, O. V, Tamm, M. V, \& Nechaev, S. K. Physical Review Letters 109, 018102 (2012).

[14] Lokhov, A. Y, Valba, O. V, Tamm, M. V, \& Nechaev, S. K. Physical Review E, 88, 052117 (2013).

[15] Friedgut, E. J. Amer. Math. Soc. 12, 1017-1054 (1999).

[16] Lando, S. K. Lectures on generating functions. (American Mathematical Society) (2003).

[17] Micali, S. \& Vazirani, V. V. In Proceedings of the 21st Annual Symposium on Foundations of Computer Science (SFCS '80), IEEE Computer Society, 17-27 (1980). 
Topological transition in disordered planar matching: combinatorial arcs expansion 28

[18] Nechaev S. K, Tamm M. V, \& Valba O. V. J. Phys. A 44, 195001 (2011).

[19] MacMahon P. A. Combinatorial Analysis, Vols. 1 and 2. (Cambridge University Press) (1915).

[20] Deutsch, E. Discrete Mathematics, 204, 167-202 (1999).

[21] Prudnikov, A. P, Brychkov, I. A, \& Marichev, O. I. Integrals and series: special functions, Vol. 2. (CRC Press) (1986)

[22] Vladimirov, A. A. Problems of Information Transmission 49, 1 (2013).

[23] Shannon, C. E. Bell System Tech. J. 27, 379 (1948). 